\title{
Failed Cell Migration and Death of Purkinje Cells and Deep Nuclear Neurons in the weaver Cerebellum
}

\author{
Stephen M. Maricich, ${ }^{1}$ Jill Soha, ${ }^{1}$ Ekkhert Trenkner, ${ }^{2}$ and Karl Herrup ${ }^{1}$ \\ ${ }^{1}$ Alzheimer Research Laboratory, Department of Neurology, Case Western Reserve University School of Medicine, \\ Cleveland, Ohio 44106, and IInstitute for Basic Research, Center for Developmental Neuroscience and Developmental \\ Disabilities, Staten Island, New York 10314
}

\begin{abstract}
The mouse neurological mutant weaver has an atrophic cerebellar cortex with deficits in both Purkinje and granule cell number. Although granule cells are known to die postnatally shortly after their final cell division, the cause of the Purkinje cell deficit (cell death vs lack of production) is unknown. We report here a quantitative analysis of large cerebellar neurons of the weaver mutant during postnatal development. We explored the hypothesis that the cells of the entire cerebellar anlage were affected by the mutation by including in our study the neurons of the deep cerebellar nuclei (DCN). Our analysis reveals that in homozygous weaver mutants (1) the DCN are displaced laterally, display an abnormal anatomy, and suffer a $20-25 \%$ decrease in neuron number; (2) this numerical deficit is located in medial regions, similar to the localization of cortical deficits in both Purkinje and granule cells; (3) pyknotic figures are present in the juvenile DCN and in the Purkinje cell layer; and (4) the
\end{abstract}

majority of cell death in these populations occurs not in medial regions where the numerical deficits are observed, but rather laterally where adult cell number is nearly normal. These results lead us to propose that the complete weaver phenotype includes a failure of the cell movements that lead to the fusion of the bilateral cerebellar anlage, and that this failure to migrate properly leaves some of the Purkinje cells and DCN neurons in a position where they are unable to make appropriate connections, leading to their death. In addition to implications for normal development, these observations suggest that weaver effects on the cerebellum can be unified into one consolidated model in which failure of cell movement affects all major cerebellar neurons.

Key words: weaver; deep cerebellar nuclei; GIRK2; ataxia; cell counts; cell death
The cerebellum is unusual among brain regions in that it spans the midline with no morphological or biochemical indications of where the right and left halves are joined. This anatomy develops from two symmetric but distinct embryonic anlage derived from the roof of the fourth ventricle. In the mouse, the growing anlage first meet at embryonic day 15 and fuse nearly completely during the ensuing 2 days. Movements of the constituent neuronal types, including Purkinje cells, granule cells, and the neurons of the deep cerebellar nuclei (DCN), have been carefully recorded (Altman and Bayer, 1985a,b,c), and disruptions of this process have been observed in two mouse mutants. The engrailed-2 knock-out mutant displays a delay in cerebellar fusion at the midline and aberrations in foliation (Millen et al., 1994), both of which may result partly from migrational failures. An even clearer picture of migrational deficits is seen in the weaver mutation, in which mutant mice possess a severely affected cerebellar structure.

weaver has been identified as a point mutation in girk2, a gene encoding a G-protein-coupled inwardly rectifying potassium channel (Patil et al., 1995). The most obvious effects of the mutation occur in the cerebellum, although other areas of the brain are also abnormal (Sekiguchi et al., 1995; Roffler-Tarlov et al., 1996). In the cerebellar cortex, there are substantial amounts of granule cell death during the first postnatal weeks of development (Rezai and

\footnotetext{
Received Dec. 24, 1996; revised Feb. 19, 1997; accepted Feb. 25, 1997.

This work was supported by a grant from National Institutes of Health (NS-20591). We thank Dr. Jerry Silver for insightful discussions concerning this manuscript.

Correspondence should be addressed to Dr. Karl Herrup, Alzheimer Research

Laboratory, Case Western Reserve University, School of Medicine E504, 10900

Euclid Boulevard, Cleveland, OH 44106.

Copyright (C) 1997 Society for Neuroscience 0270-6474/97/173675-09\$05.00/0
}

Yoon, 1972; Rakic and Sidman, 1973; Smeyne and Goldowitz, 1989). Most of this cell loss occurs before the granule cells complete migration to the internal granule cell layer. Approximately $40 \%$ of the Purkinje cells are also missing from the adult cerebellum (Herrup and Trenkner, 1987), although it is not clear whether this results from cell death or a failure of genesis. All of the phenotypes vary from region to region in the cerebellar cortex. Granule cells in the hemispheres migrate and survive in greater numbers than near the midline (Herrup and Trenkner, 1987). Purkinje cell number is also nearly normal in the hemispheres, with the majority of the deficit being observed medially (Rezai and Yoon, 1972; Blatt and Eisenman, 1985; Herrup and Trenkner, 1987). This mediolateral distribution of effects led Herrup and Trenkner (1987) to speculate that midline fusion of the cerebellar anlagen is affected in weaver. If this were true, one might also expect to find some disruption of other cerebellar components, such as the DCN.

The DCN, along with the vestibular nuclei, constitute the sole output of the cerebellum. Most anatomists agree on a scheme that divides these nuclei into three major divisions: medialis, interpositus, and lateralis. Cortical input to the DCN is supplied via Purkinje cell axons. Anterograde tracing and degeneration studies (Jansen and Brodal, 1940; Armstrong and Schild, 1978a,b) demonstrate the following basic arrangement of cortico-nuclear connections: medialis receives Purkinje cell input from the vermis, interpositus from the intermediate hemispheres, and lateralis from the lateral hemispheres. Despite the detailed knowledge of cortico-nuclear circuitry, the mechanisms controlling neuronal cell number in this population of neurons have remained elusive. 
Table 1. Uncorrected and corrected neuron numbers in the different constituent nuclei of the DCN

\begin{tabular}{|c|c|c|c|c|c|c|c|c|}
\hline & \multicolumn{3}{|l|}{ C57BL/6J } & \multicolumn{5}{|l|}{ B6CBA } \\
\hline & $\begin{array}{l}\text { Wild type } \\
\text { count (SEM) }\end{array}$ & $\begin{array}{l}w v / w v \\
\text { count (SEM) }\end{array}$ & $\% \mathrm{wt}$ & $\begin{array}{l}\text { Wild type } \\
\text { count (SEM) }\end{array}$ & $\begin{array}{l}+/ w v \\
\text { count (SEM) }\end{array}$ & $\% \mathrm{wt}$ & $\begin{array}{l}w v / w v \\
\text { count (SEM) }\end{array}$ & $\% \mathrm{wt}$ \\
\hline \multicolumn{9}{|c|}{ Uncorrected counts } \\
\hline Lateralis & $4550( \pm 166)$ & $4930( \pm 670)$ & 108.4 & $3750( \pm 323)$ & $4610( \pm 320)$ & 123.0 & $3970( \pm 139)$ & 106.0 \\
\hline Interpositus & $11200( \pm 693)$ & $8660( \pm 1070)$ & 77.3 & $8980( \pm 411)$ & $9670( \pm 404)$ & 107.6 & $6570( \pm 560)$ & 73.2 \\
\hline Medialis & $5910( \pm 208)$ & $5300( \pm 485)$ & 89.7 & $5430( \pm 311)$ & $5960( \pm 44)$ & 109.6 & $4010( \pm 132)$ & 73.8 \\
\hline Total & $21800( \pm 411)$ & $17700( \pm 1124)$ & 81.2 & $18200( \pm 373)$ & $20200( \pm 548)$ & 111.4 & $14600( \pm 614)$ & 80.2 \\
\hline \multicolumn{9}{|c|}{ Corrected counts } \\
\hline Lateralis & $2750( \pm 100)$ & $2910( \pm 395)$ & 105.8 & $2380( \pm 222)$ & $2740( \pm 193)$ & 115.1 & $2360( \pm 150)$ & 98.9 \\
\hline Interpositus & $7080( \pm 436)$ & $5250( \pm 649)$ & 74.2 & $5550( \pm 275)$ & $5460( \pm 295)$ & 98.5 & $* 3860( \pm 168)$ & 69.7 \\
\hline Medialis & $3690( \pm 130)$ & $2940( \pm 269)$ & 79.7 & $3520( \pm 208)$ & $3500( \pm 73)$ & 99.4 & $* 2350( \pm 118)$ & 66.8 \\
\hline Total & $13400( \pm 274)$ & $* 10500( \pm 658)$ & 78.0 & $11400( \pm 257)$ & $11700( \pm 405)$ & 102.2 & $* 8570( \pm 172)$ & 74.9 \\
\hline \multicolumn{9}{|c|}{ \# Brains (\# sides) } \\
\hline Coronal & $2(3)$ & $1(2)$ & & $2(4)$ & $2(4)$ & & $2(4)$ & \\
\hline Sagittal & $1(1)$ & $1(2)$ & & & & & & \\
\hline
\end{tabular}

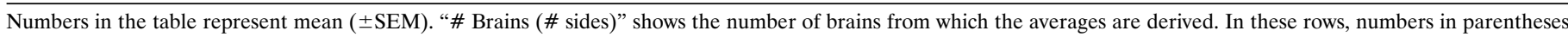

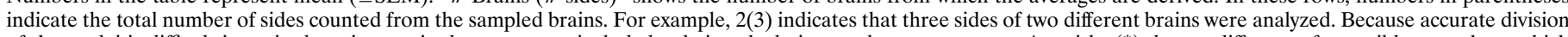

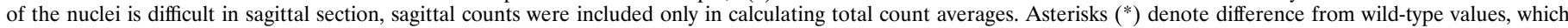
is statistically significant at the $95 \%$ confidence level (ANOVA, Newman-Keuls; $p<0.05$ ).

It seems that the DCN are numerically unaffected in many of the mouse mutants with strong phenotypes in cerebellar cortex. Only two mutants unequivocally demonstrate cell deficiencies in the deep nuclei: Purkinje cell degeneration (Triarhou et al., 1987) and vibrator (Weimer et al., 1982). To date, no spatial matching of numerical disruptions of cortex and deep nuclei have been reported.

We present data demonstrating an effect of the weaver mutation on the morphology and cell number of the DCN. This effect seems to be localized medially, roughly corresponding to the medial deficits seen in both granule cell and Purkinje cell number. We also present evidence for increased cell death in the DCN during development of weaver mice. Finally, analysis of Purkinje cell plus Golgi type-II cell number in the cerebellar cortex demonstrates that weaver mice begin postnatal life with a normal complement of Purkinje cells that are subsequently lost during the third postnatal week.

\section{MATERIALS AND METHODS}

Animals. C57BL/6J wild-type and $w v / w v$ brains were obtained from our slide archives. Stocks of all B6CBA mice were obtained from The Jackson Laboratory (Bar Harbor, ME). DCN neuron counts of wild-type mice of each strain [C57BL/6J: postnatal day (P) ages P62, P164, and P177; B6CBA: ages P54 and P58] were used as controls. Homozygous $w v / w v$ mice were also of each strain background (C57BL/6J: ages P38 and P40; B6CBA: ages P40 and P114). Heterozygous $+/ w v$ mice (both P148) were on the B6CBA background. Medium-to-large neuron (MLN) counts were performed on C57BL/6J mice of the following ages: (wild-type) P10, P16, $\mathrm{P} 24$, and (weaver) P9, P12, P14, P19. Genotypes of wild-type and heterozygous mice were determined by examination of cerebellar morphology; homozygous mutant mice were identified by ataxic phenotype.

Perfusion and histology. All mice were anesthetized deeply with Avertin (20 $\mu \mathrm{l} / \mathrm{gm}$ body weight) and perfused transcardially with $4 \%$ paraformaldehyde in $0.1 \mathrm{M}$ phosphate buffer. Brains were dissected and post-fixed in the same solution overnight. Overnight equilibration in $1 \times$ PBS was performed before embedding.

Embedding in paraffin was performed by the Case Western Reserve University Neuropathology unit. Serial $8 \mu \mathrm{m}$ microtome sections were cut, and a careful record of lost sections (typically two to three per brain) was kept to determine accurate distances. One wild-type and one weaver brain were sectioned sagittally; the remaining nine brains were sectioned coronally. Sections were rehydrated, stained with a $0.01 \%$ cresyl violet solution, dehydrated, treated with Permount, and coverslipped.
Cell counts and correction factors. Before counting, the morphology of the deep nuclei was followed rostrocaudally (coronal sections) or mediolaterally (sagittal sections) under a dissecting microscope to insure accurate parcellation of cell groups. Cell counts of both DCN neurons and medium-to-large cortical neurons were performed at $400 \times$. DCN neuron counts were taken from sections spaced $\sim 80 \mu \mathrm{m}$ apart, whereas MLN neurons were counted in sections spaced $160 \mu \mathrm{m}$ apart; there was $\leq 5 \%$ intra- and inter-observer variability. Criteria for counting included neuronal morphology (clear nucleus, prominent nucleolus, Nissl substance) and the presence of a clear nuclear outline.

Cell and nuclear diameters and areas were obtained using the Eutectic Electronics Neuron Tracing System (Raleigh, NC). Briefly, outlines of cells and their nuclei were made in three sections (rostral, intermediate, and caudal for transverse series; medial, intermediate, and lateral for sagittal series) for each brain, and the Eutectics statistics software was used to calculate diameters and areas. Data were transferred to a Microsoft Excel (Microsoft) spreadsheet written to calculate correction factors according to the method of Hendry (1976). Correction factors were computed separately for each of the nuclei, and at least 300 cells/brain were measured; at least 200 MLNs were measured to calculate cortical correction factors.

Analysis of cell death. Sagittal cresyl violet-stained sections of immature C57BL/6J mice were observed at $400 \times$ for the presence of dying cells and pyknotic nuclei. Wild-type mice ages $\mathrm{P} 13, \mathrm{P} 16, \mathrm{P} 17$, and $\mathrm{P} 25$, and $w v / w v$ mutants ages P14, P19, and P25 were examined. One animal of each age was obtained from our slide archives. Two coronally sectioned P17 B6CBA weaver brains were also examined.

Statistics. All count and percentage data obtained for the DCN were entered into Microsoft Excel and analyzed with the Analysis Tools Toolpak. One-way ANOVA was used to compare mean counts and mean percentage composition values both among and between strains. Pairwise population differences were determined using the Newman-Keuls procedure.

\section{RESULTS}

\section{Effects of the weaver mutation on deep nuclear size and morphology}

The gross overall morphology of the DCN appears similar in both B6CBA and C57BL/6J mice (Fig. 1). In all mice examined, the basic divisions of the DCN could be identified. The caudalmost nuclear division seen in both strains is nucleus medialis rather than nucleus interpositus as reported in rat by Korneliussen (1968). This variation is likely to be caused by interspecific differ- 


\section{B6CBA}
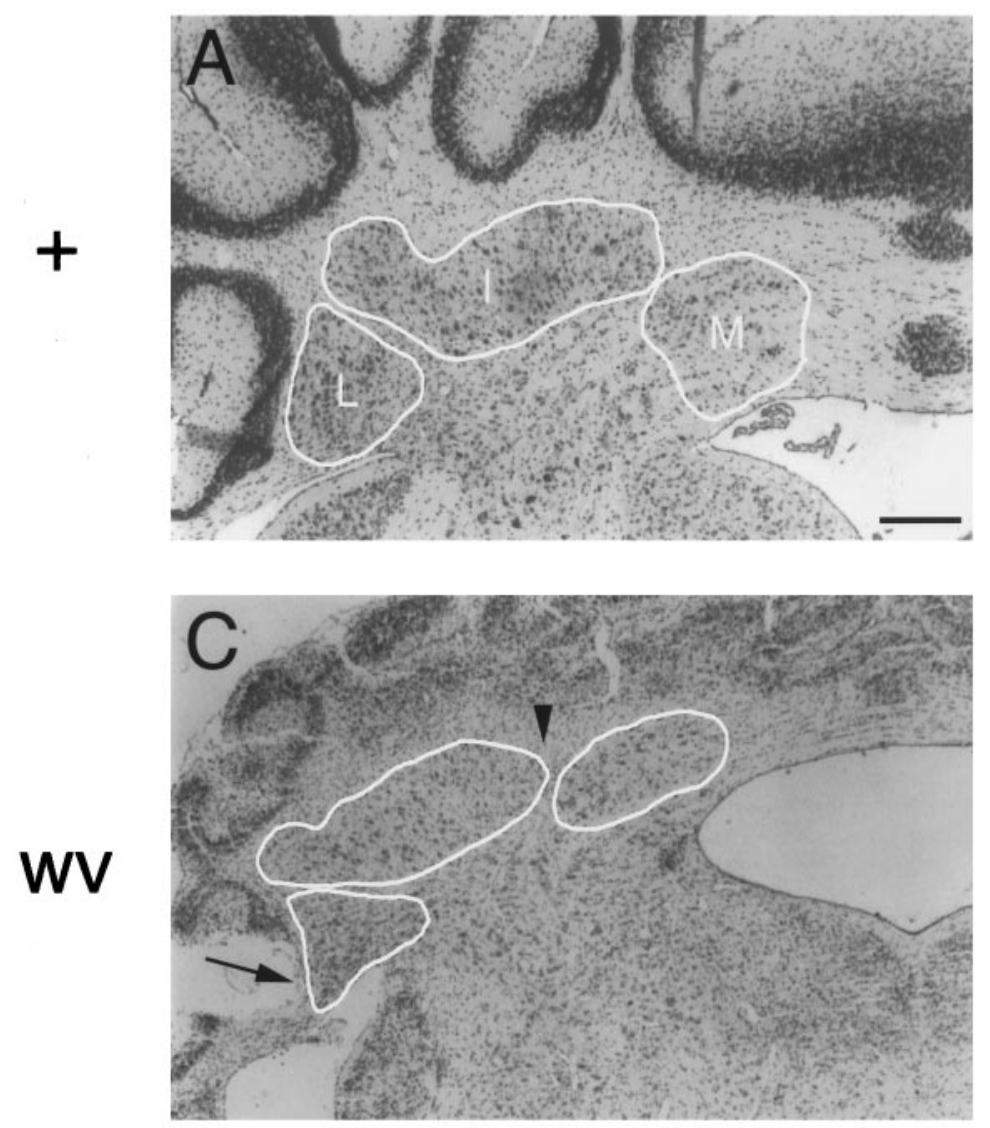

\section{C57BL/6J}
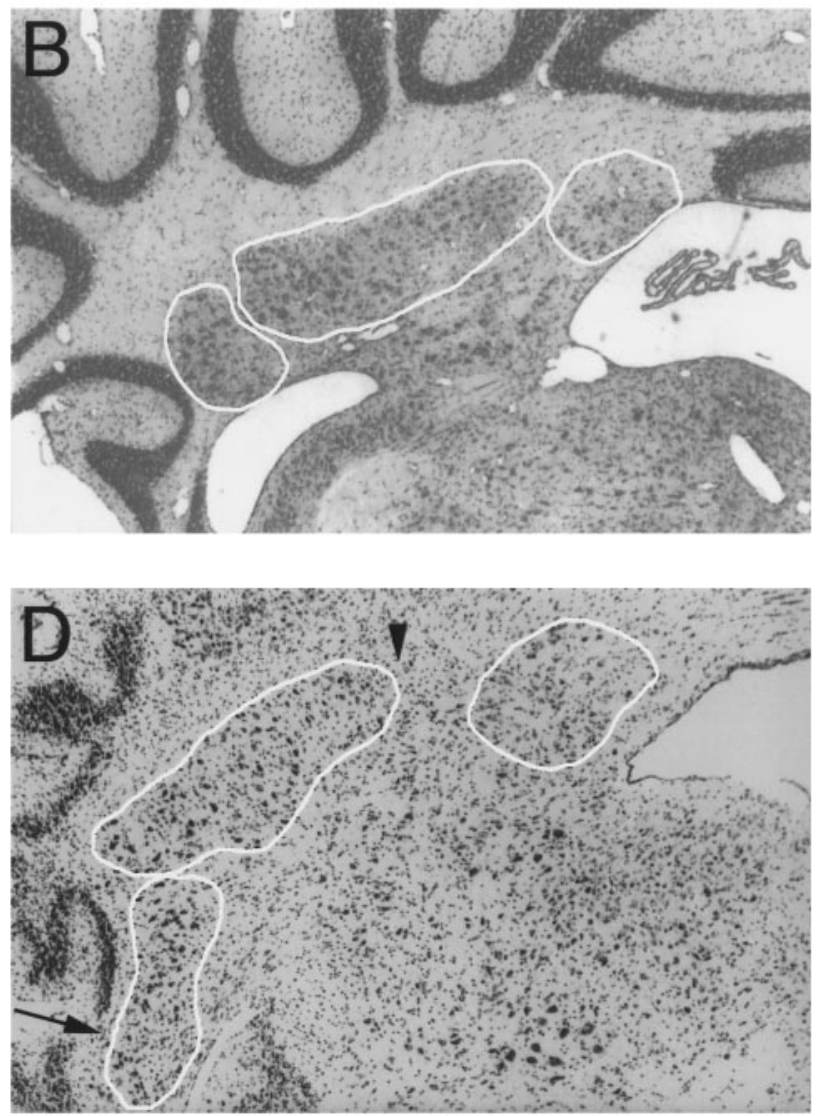

Figure 1. The weaver mutation affects the basic morphology of the DCN in C57BL/6J and B6CBA mouse strains. All photographs are at 50 $\times$ magnification from approximately the same rostrocaudal level of adult animals; individual nuclei are outlined and indicated on each photo. $A$, B6CBA $+/ w v$ heterozygote. Wild-type B6CBA DCN (not shown) appear virtually identical to the DCN of the heterozygote. $B$, Wild-type C57BL/6J DCN. Note that nucleus interpositus is slightly more elongated compared with B6CBA DCN. $C$, B6CBA wv/wv mutant. Arrows point to nucleus lateralis neurons in the white matter of the paraflocculus, a condition that is absent in wild-type and heterozygous mice. Arrowheads illustrate the more lateral location of the medial boundary of nucleus interpositus. $D, \mathrm{C} 57 \mathrm{BL} / 6 \mathrm{~J} w v / w v$ mutant. Although larger in absolute size than the B6CBA homozygous mutant, the basic DCN anatomy is unaltered. Arrows and arrowheads same as $C$. Scale bar, $200 \mu \mathrm{m}$.

ences that should not affect our ability to classify cells into the separate nuclei. The only anatomical difference we detected between mouse strains is the slightly more elongated appearance of nucleus interpositus in the C57BL/6J strain.

On both strain backgrounds, $w v / w v$ mutants display a mediolateral shortening of nucleus interpositus (Fig. 1C,D) when compared with wild-type or heterozygous mice (Fig. $1 A, B$ ). Cells of the lateral nucleus can be found occupying white matter in the paraflocculus of the homozygous mutants (arrows, Fig. 1C,D), but not of wild-type or heterozygous mice (Fig. $1 A, B$ ). In addition, the medial aspect of nucleus interpositus is located more laterally in the cerebellum of $w v / w v$ mice (arrowheads, Fig. 1C,D). In all genotypes the rostrocaudal length of the nuclei increases with age from P40 to P177; controlling for age, significant differences in rostrocaudal dimensions were not observed. In sagittal section, the mediolateral extent of the DCN was shortened by $\sim 250 \mu \mathrm{m}$ in the C57BL $/ 6 \mathrm{~J} w v / w v$, representing a $13 \%$ decrease compared with wild type.

\section{Cell number in the DCN}

Uncorrected and corrected cell counts are summarized in Table 1. Correction factors (Hendry, 1976) were calculated separately for each nucleus at three levels of each brain in coronal section; correction factors were calculated medially, intermediately, and laterally for sagittally sectioned brains. We observed a $>20 \%$ decrease $(22 \%$ C57BL/6J, 25\% B6CBA) in the total number of neurons present in the deep nuclei of homozygous mutants on both strain backgrounds when compared with wild-type controls of the same strain. Moreover, the deficit can be accounted for almost completely by losses in the interpositus and medialis nuclei, whereas nucleus lateralis appears to be spared. Nucleus interpositus loses $\sim 30 \%$ of its neurons in both strains, whereas the losses in nucleus medialis differed on different strain backgrounds (20\% C57BL/6J, 33\% B6CBA). All cell number differences between wild type and mutant (total counts and individual nuclei) on the B6CBA strain background are statistically significant at the $95 \%$ confidence level. The reduction in total cell number is statistically significant on the C57BL/6J strain background. DCN counts in B6CBA heterozygous $(+/ w v)$ animals were also compared with wild-type neuron numbers; ANOVA revealed no significant differences at the $95 \%$ confidence level. Figure 2 shows corrected neuron counts graphed as a percentage of wild-type numbers. 
Figure 2. The weaver mutation selectively affects medial portions of the DCN, causing a decrease in total neuron number. Neuron counts are graphed as a percentage of wild type on the same strain background; absolute numbers for these animals are presented in Table 1. Asterisks denote difference from wildtype values that is statistically significant (ANOVA, Newman-Keuls; $p<0.05$ ). Error bars represent SEM.

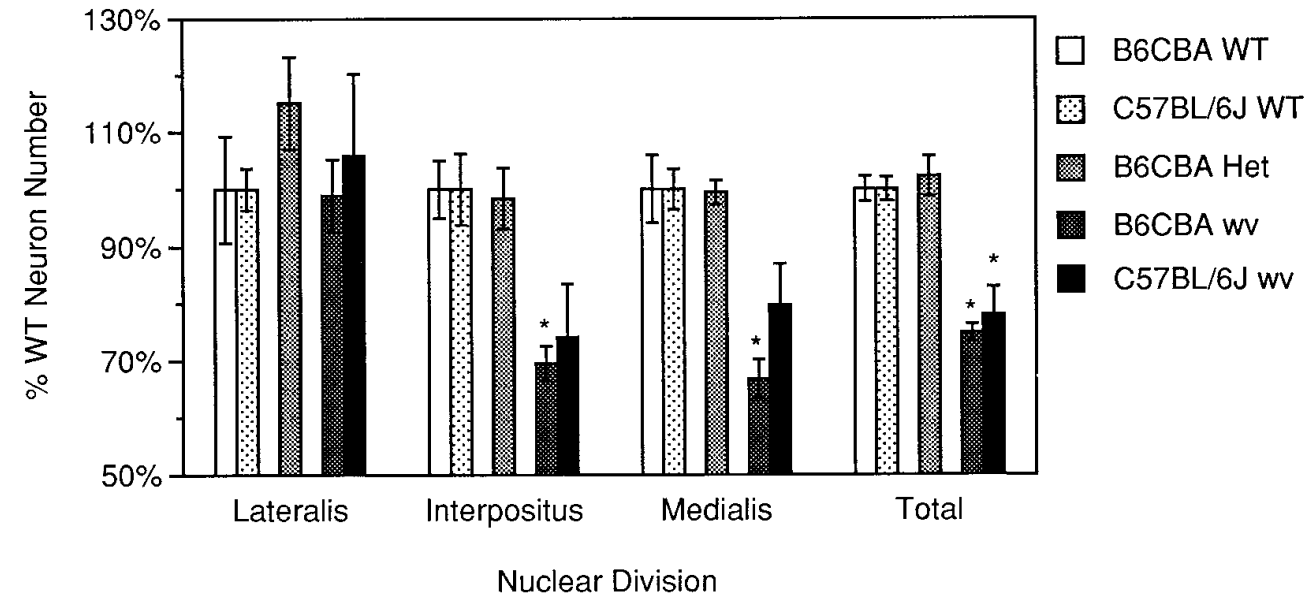

Figure 3. Comparison of DCN neuron distribution in $\mathrm{C} 57 \mathrm{BL} / 6 \mathrm{~J}$ wild type and weaver. Cell counts were taken every $80 \mu \mathrm{m}$ in sagittally sectioned brains and then graphed versus percentage mediolateral distance from the most medial aspect of the DCN. One side of one wild-type and both sides of one weaver brain are shown. Note the selective decrease in medial counts, particularly those in medial nucleus interpositus, in the weaver DCN. Bars at the top of the figure indicate approximate boundaries of each nucleus as determined by measurement of nuclei in several coronal sections.

Genetic background has a substantial impact on cell number, as noted in previous studies (Herrup, 1986; Herrup and Sunter, 1986). Total deep nuclear cell number differs by approximately one sixth between B6CBA and C57BL/6J (ANOVA, $F=20.6$, $p<10^{-5}$; Newman-Keuls, $\left.p<0.05\right)$. These differences most likely represent true interstrain differences. There is no significant difference, however, in the relative percentage composition of the nuclei in genotype-matched animals from the two strain backgrounds (data not shown).

Figure 3 compares the distribution of DCN cell counts in sagittal sections for adult $\mathrm{C} 57 \mathrm{BL} / 6 \mathrm{~J}$ wild-type and weaver deep nuclei. The number of cell profiles in most sections is lower in $w v / w v$ than in wild type, and the majority of the decrease is found in the region that most closely approximates nucleus interpositus. Although graphing the counts versus percentage of mediolateral distance demonstrates that the peaks representing nucleus medialis and nucleus lateralis are both present in the mutant, the absolute distance between them is less than that seen in wild-type

cerebella (data not shown) (compare Fig. 1, $C$ and $D$ with $A$ and $B$ ). Parsing the nuclei using mediolateral dimensions obtained in coronal section (bars, Fig. 3) results in deficits in cell number of $2 \%$ laterally, $34 \%$ in the intermediate region, and $15 \%$ medially. These are not absolute measures of cell number in medialis, interpositus, and lateralis, because there is some overlap of these nuclei in sagittal section; however, the same trend seen in the individual nuclei in coronal section of $\mathrm{C} 57 \mathrm{BL} / 6 \mathrm{~J}$ cerebella is also seen moving laterally from the midline in the sagittal series.

It should be noted that the DCN from the right side of one C57BL/6J $w v / w v$ brain closely approximated wild-type corrected cell numbers $(12,400$ cells). This asymmetry and high cell number were not observed in any of the other mutants examined; however, the cell distribution was similar to that of the weaver mutant (greater percentage of cells found laterally compared with wild type). This raises the possibility that DCN cell number control is independent on each side of the cerebellum (Herrup et al., 1984) and that the weaver mutation is not necessarily fully penetrant on both sides of the same brain.

\section{Cell death}

To determine whether cell death plays a role in the decreased deep nuclear neuron numbers seen in adult weaver mice, the presence of pyknotic nuclei was assessed in sagittal sections of C57BL/6J wild-type and homozygous mutants at various postnatal ages. There were no obvious differences in the number of pyknotic nuclei seen in the two genotypes before P13; however, at ages beyond this time point, a clear increase in the number of dying cells was apparent in weaver mutants. Pyknoses are obvious and common in juvenile weaver DCN, as demonstrated by the P14 cerebellum shown in Figure $4 A, B$. Figure 5 charts the number of pyknoses seen in the DCN at four wild-type and three mutant postnatal ages. Although direct comparisons are difficult given the age variation, it is easy to appreciate the dramatic increase in pyknoses seen in the weaver brains compared with wild type. The number of pyknotic nuclei found in the lateral half of the cerebellum is greater than that seen in the medial half in sagittal sections; this suggests that most neuronal death occurs in the lateral portion of nucleus interpositus and throughout nucleus lateralis. This observation is especially curious because cell counts in adult mice reveal a nearly normal number of cells in nucleus lateralis (Table 1, Figs. 2, 3). Most cell death in the weaver is observed around P19, with less found at P25. In addition, there appears to be a shift in the site of cell death toward more lateral regions as weaver mice age. Pyknosis in the wild type is much rarer 

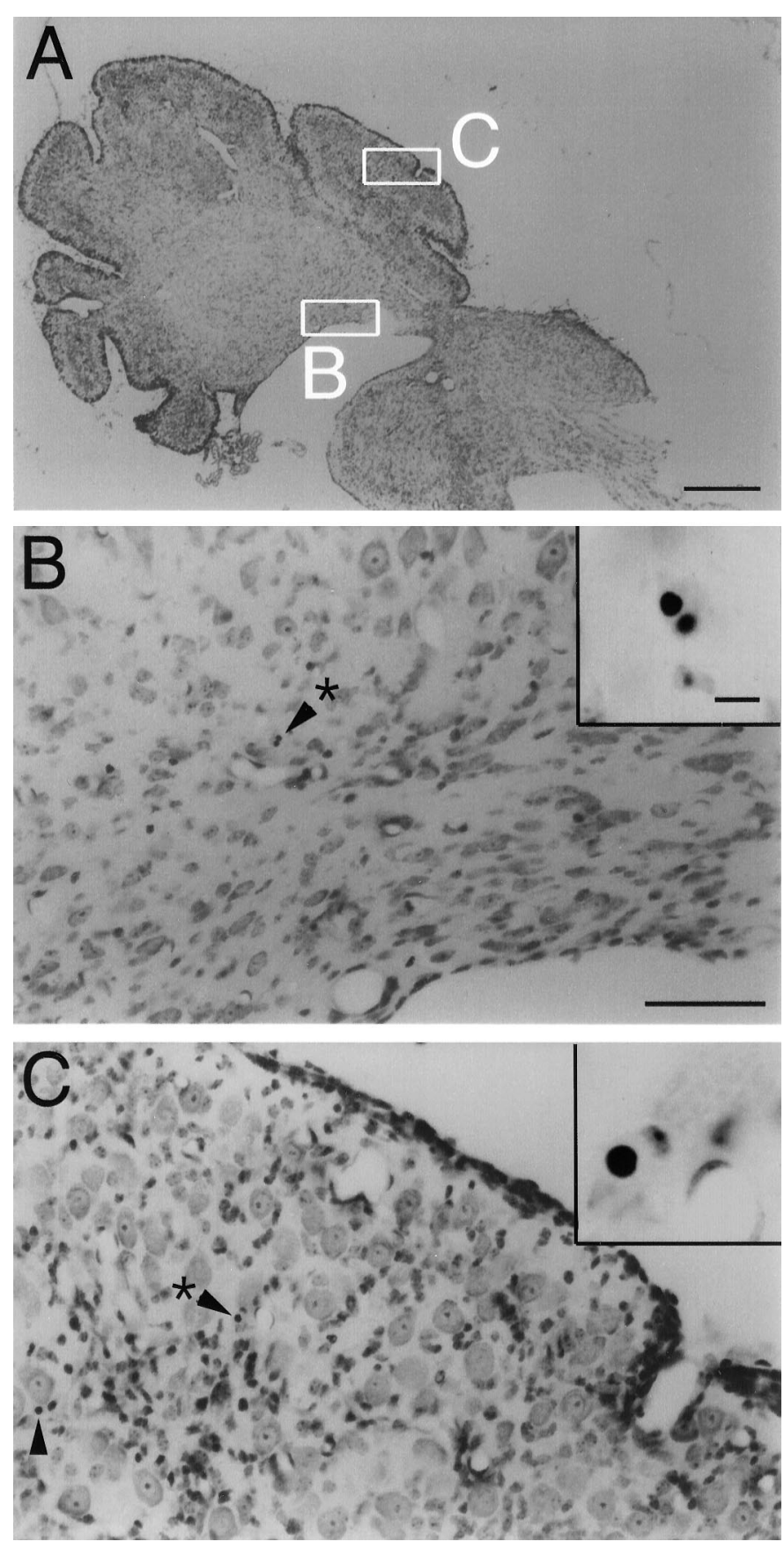

Figure 4. Juvenile weaver mutants contain dying cells in the DCN and Purkinje cell layer. Photographs are of one lateral section of a C57BL/6J P14 weaver cerebellum sectioned sagittally. Rostral is to the right in all photographs. $A$, Lateral cerebellum at $50 \times$. White boxes correspond to views in $B$ and $C$. $B, \mathrm{DCN}$ at $400 \times$. Arrowhead and asterisk indicate a dying cell seen at $1000 \times$ in inset. $C$, Cerebellar cortex at $400 \times$. Two putative pyknotic Purkinje cells are indicated by arrowheads; inset is $1000 \times$ photo of cell marked by asterisk. Pyknotic Purkinje cells were not observed in wild-type cortex at any age (data not shown). Scale bars: $A, 200 \mu \mathrm{m} ; B, C$, $50 \mu \mathrm{m}$; insets, $5 \mu \mathrm{m}$.

and tends to be distributed more evenly across the nuclei (Fig. $5 A$ ). Pyknoses were also seen in $\mathrm{P} 17 \mathrm{~B} 6 \mathrm{CBA} w v / w v$ brains cut in coronal section ( $n=2$, data not shown). No pyknotic nuclei were observed in adult material of any of the animals examined, regardless of genotype.

The presence of a deficit in adult Purkinje cell numbers

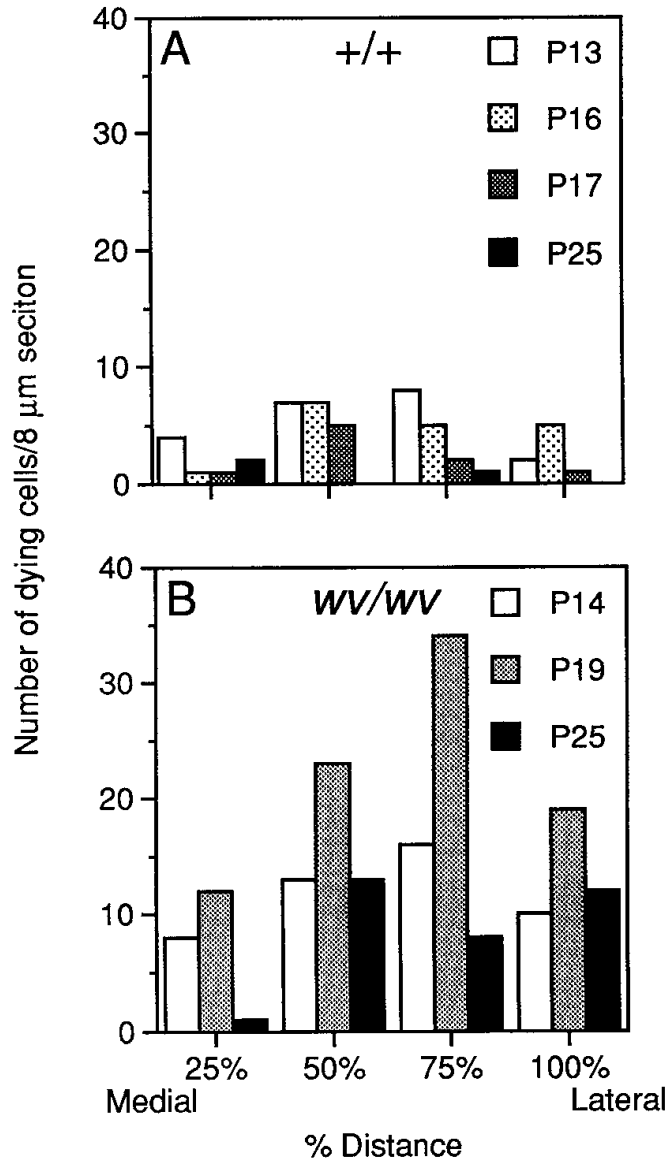

Figure 5. Number of pyknoses in the DCN of juvenile weaver mutants is higher than that found in wild-type mice. The number of dying cells was determined for C57BL/6J animals every $80 \mu \mathrm{m}$ in sagittally sectioned brains (one side of one brain of each age). The DCN was then divided into four regions of equal size by percentage of total mediolateral distance, and the counts were summed for each of these regions. $A$, Wild-type DCN pyknoses. Dying cells are relatively rare and evenly distributed across the mediolateral extent of the DCN. B, weaver DCN pyknoses. Pyknotic figures are seen in greater numbers in lateral portions of the DCN. The largest number of dying cells is seen at $P 19$.

prompted us to examine cell death in the cerebellar cortex as well. Although there is substantial granule cell death in this area, we looked specifically in the Purkinje cell layer for the presence of large pyknotic nuclei surrounded by cytoplasmic remnants that might betray their identity as dying Purkinje cells. Virtually no such figures are found in wild-type mice (ages P13, P16, and P17 examined; data not shown). Pyknotic material, however, is present in the Purkinje cell layer of weaver cerebellar cortex, particularly in the lateral regions (Fig. 4C). The presence of pyknosis in the granule cell layer made it impossible to accurately quantitate the numbers of dying Purkinje cells. Thus, a different approach was necessary.

\section{Cell number in the cerebellar cortex}

To address further the question of whether the deficit in Purkinje cell numbers seen in the weaver cerebellar cortex was attributable to cell death, we directly determined Purkinje cell numbers at different postnatal ages. Because it is difficult to define a precise Purkinje cell layer in the mutant, it is difficult to unequivocally identify Purkinje cells in cresyl violet-stained sections. Accordingly, MLN number, which should include both Purkinje cells and 


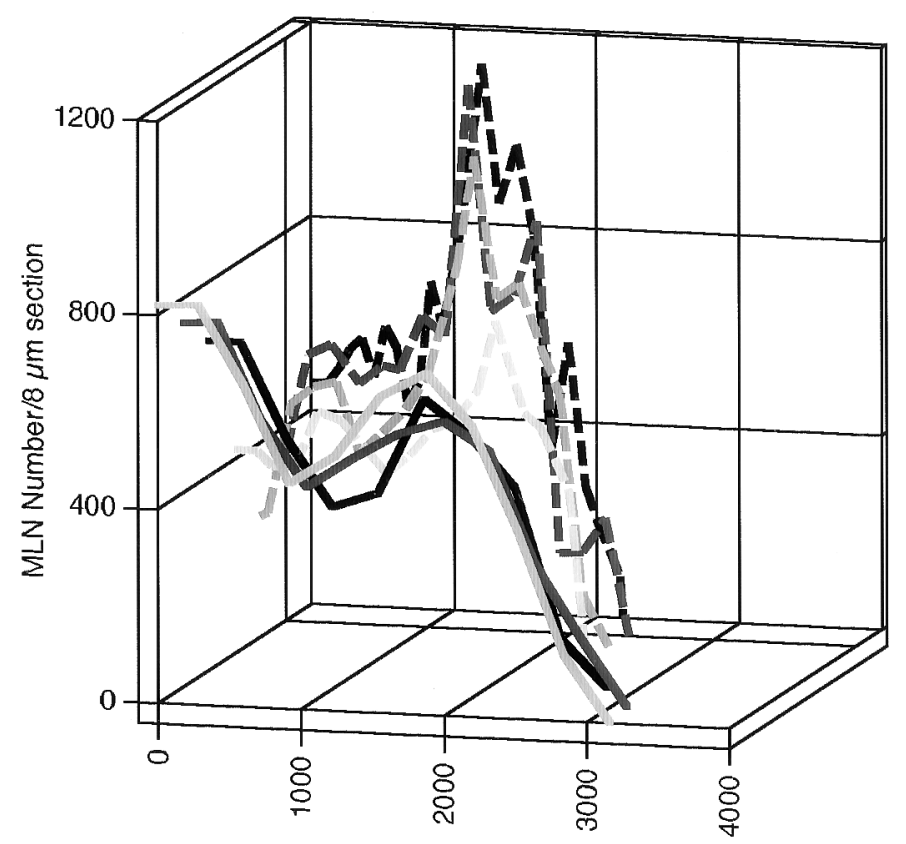

Mediolateral Distance $(\mu \mathrm{m})$

\begin{tabular}{|rrrr|}
\hline Wildtype & Weaver & & \\
P24 & P19 & P12 \\
P16 & P14 & $=$ & P9 \\
P10 & & & \\
\hline
\end{tabular}

Figure 6. Medium-to-large neuron $(M L N)$ number decreases laterally in weaver mutants between P9 and P19. The number of MLNs was counted every $160 \mu \mathrm{m}$ in sagittal sections of C57BL/6J cerebella and graphed versus distance from the midline. Counts from one side of three wild-type animals (solid lines) and four weaver cerebella (dotted lines) are shown. At $\mathrm{P} 9$, weaver mice have many more MLNs laterally than do wild-type mice. These numbers gradually decline toward wild-type levels by P19. No change takes place in medial MLN numbers.

Golgi type-II cells (Herrup and Mullen, 1979), was determined in sagittal sections of C57BL/6J cerebella.

Figure 6 demonstrates MLN count distributions in sagittal section for wild-type and $w v / w v$ mutants (one side of one animal of each age). The relative distribution of MLNs in the wild-type cortex remains the same, regardless of age. Cell counts from individual sections near the midline average $\sim 750$ and vary from 680 to 820 , whereas lateral cell counts do not exceed 710 cells/ section. These results are dramatically different from those seen in the weaver mice. First, at all ages the medial counts are lower than those for wild type, varying between 250 and 550 and centering around 400. Second, peak counts found laterally before P20 are higher at all ages than those seen in wild-type cerebella. This effect is largest at early ages (P9 and P12; Fig. 6, darkest lines), decreasing toward wild-type levels by P19. It has been shown previously that the peak lateral MLN counts are nearly the same for adult wild-type and weaver mice (Herrup and Trenkner, 1987).

Figure 7 graphs total MLN number in weaver cortex as a percentage of wild-type number at equivalent ages. Total MLN counts approximate those seen in wild-type animals until P14. Between P14 and P19 there is a sharp decrease in MLN number seen in the weaver mutant. The P19 value of $75 \%$ wild-type MLN number is intermediate to that seen at P14 (100\%) and P30 (60\%; Herrup and Trenkner, 1987). Thus, it appears that $w v / w v$ mutant

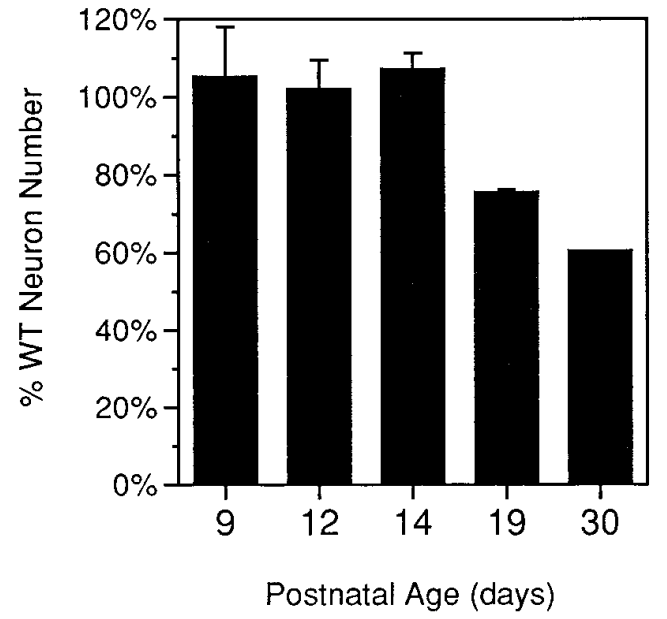

Figure 7. Total MLN number decreases from wild-type levels as weaver animals age. Mean MLN counts (average of both sides of the same brain) are graphed as a percentage of wild-type counts at corresponding ages; P9-P19 brains are the same as those shown in Figure 6. Error bars represent SEM. Data for the P30 weaver were obtained from Herrup and Trenkner (1987).

animals are born with a full complement of Purkinje cells and that they lose these cells during the subsequent stages of cerebellar maturation.

A similar analysis of DCN neuron numbers during the first two postnatal weeks was not possible because of the difficulty in accurately identifying nuclear borders and assigning neurons to the DCN, especially in weaver mutants.

\section{DISCUSSION}

The data presented here expand our understanding of the effects of the weaver mutation in several important ways. First, we have added an additional cell type to the list of those affected in the homozygous $w v / w v$ mouse. The DCN are abnormal in both their overall anatomy and constituent neuron number. The magnitude of the cell loss, $20-25 \%$ of total DCN neurons, is independent of genetic background (B6CBA or $\mathrm{C} 57 \mathrm{BL} / 6 \mathrm{~J}$ ), even though there are clear strain differences in absolute neuron numbers. Second, we have shown that the cell loss in the mutant DCN is regionally variable, with a lateral-to-medial gradient of increasing severity. This regional pattern of cell loss is identical to that seen in the cerebellar cortex in both the granule cell and Purkinje cell populations, suggesting a consistent action of the mutation throughout the weaver cerebellum. Third, the observation of numerous pyknotic figures in the weaver DCN and cerebellar cortex, as well as quantitative analysis of MLN number at various postnatal ages, demonstrates that the deep nuclear and Purkinje/Golgi II cell deficits result from neurodegeneration (in the third postnatal week) rather than from a failure of neurogenesis. Finally, we have shown, in both cortex and DCN, that cell death occurs not in the medial regions where the cells are missing in the adult but rather in the more lateral portions where cell number is nearly normal.

\section{An expanded model of the weaver mutation}

The involvement of the DCN in the weaver phenotype emphasizes the need to consider all of the cerebellar cell types, not just the granule cell, as primary targets of the mutant gene. Although the flow of information in the cerebellar circuit (granule cell $\rightarrow$ Purkinje cell $\rightarrow$ deep nucleus) might imply that many of the defects in the $w v / w v$ mouse are retrograde and target-related, 
perhaps initiating in the DCN, this scenario seems unlikely because both granule cells and Purkinje cells die in $+/ w v$ heterozygotes in the absence of DCN neuron death. Equally unlikely is an anterograde effect beginning with loss of granule cells, because $\mathrm{x}$-irradiation of the granule cell layer during early postnatal development causes loss of virtually all granule cells, yet has no reported effect on Purkinje or DCN cell number (Altman and Anderson, 1972; Mariani et al., 1990). We suggest that the mutation in girk 2 operates in a cell-autonomous manner in each of these neuronal populations. The absence of DCN phenotypes in heterozygous mice suggests that unlike some Purkinje cells and granule cells, DCN neurons do not require two functional copies of the girk2 gene to be viable, although complete absence of the wild-type gene product seems to be detrimental.

By both quantitative (Figs. 5, 6, 7) and qualitative (Fig. 4B,C) means, we have demonstrated that the deficits of DCN neurons and Purkinje/Golgi II cells in weaver result from cell death rather than failed genesis. The timing of this death is intermediate between the earlier onset Purkinje cell loss in the lurcher mutant and the later occurring cell death reported in nervous and Purkinje cell degeneration (Sidman and Green, 1970; Caddy and Biscoe, 1979; Triarhou et al., 1987). It is significant that the latest reported expression of the weaver gene (girk2) in Purkinje cells is at P7 (Slesinger et al., 1996), and that GIRK2 protein is absent in this population at P19 (Liao et al., 1996). Because we do not observe Purkinje cell death until the second postnatal week (Fig. 7), it seems unlikely that these deaths are caused directly by the presence of the defective wvGIRK2 subunits. Rather, our results emphasize the importance of the ectopia. Expression of girk2 in the developing neurons of the DCN has not been reported specifically, probably because of the difficulty of unequivocally identifying these neurons at embryonic time points. girk2 is expressed throughout the developing cerebellar anlage at E14.5 (Slesinger et al., 1996), at which time all DCN neurons are postmitotic and migratory (Taber Pierce, 1975).

Given the lateral-to-medial gradient of cell loss, it was unexpected that cell death would be more extensive in the lateral half of the cerebellum. Superficially this observation seems to contradict the medial cell number decrement observed in adults. We propose that these findings suggest a "failed fusion" model of weaver gene action. On the basis of the distribution of MLN counts in adult animals, Herrup and Trenkner (1987) suggested that the joining of the cerebellar anlage might be impeded in $w v / w v$ mutants. The developmental patterns of cell loss reported here support and extend this conclusion. We suggest that a significant fraction of the large cerebellar neurons are impeded in a lateral-to-medial component of their movement and therefore are found ectopically in more lateral regions of the $w v / w v$ cerebellum. Here, either because they are ectopic per se or because they are unable to make appropriate connections, they subsequently die; this fate is well established for supernumerary (Oppenheim, 1991) or ectopic neurons (Clarke and Cowan, 1976). As would be predicted from this perspective, evidence of cell death (pyknosis) is found in the lateral portion of the cerebellum. Our quantitative analysis of MLN number in the cerebellar cortex further supports this interpretation. Wild-type and mutant mice have equal numbers of Purkinje/Golgi II cells during the first 2 postnatal weeks, but the spatial distribution of these neurons is very different. In wild-type mice, the highest cell counts are found medially, whereas in weaver the highest counts are found in the hemispheres. Based on the changes in number that occur between P9 and P19 (Fig. 6), it is the supernumerary neurons in the hemispheres that die. Our data unify weaver cerebellar defects into a cohesive model that begins with cell movement deficits and ends in cell death.

Possible causes of death of these neurons include aberrant efferent and afferent targeting. Tracing studies in the rat (Shirasaki et al., 1995) have demonstrated that axons leaving the developing cerebellar plate project caudally at early time points (E15) and rostrally later (E16 and after). Interestingly, in adult rats the main projection site of nucleus lateralis is the red nucleus (found rostral to the cerebellum), whereas nucleus medialis has a substantial projection to the spinal cord and locus coeruleus (both found caudally). Perhaps DCN neurons that settle ectopically secondary to a failure in migration are forced to follow incorrect guidance cues attributable to their ectopic location, and thus project to incorrect sites. This mismatching of axons and their targets may be responsible for the observed cell death. In addition, it is likely that there is incorrect climbing fiber and mossy fiber input to both the DCN and Purkinje cells. It has been demonstrated that climbing fibers grow into the cerebellar plate before the completion of DCN neuron and Purkinje cell movement (Bayer and Altman, 1996). Presumably, disruption of cell movements would result in disruption of these connections as well, and afferent input has been demonstrated to be an important factor for neuronal survival (Linden, 1994). Alternatively, as suggested by Clarke and Cowan (1976), the ectopia itself might be deleterious.

Although previous observations of the effect of the weaver mutation in the substantia nigra do not fit easily into this scenario, there are aspects of the phenotype in the weaver midbrain that lend support to our hypothesis. First, although they appear to migrate properly to their adult positions, subtle disruptions of cell position might be more difficult to observe in this region of the brain. Dopaminergic neurons in the substantia nigra do have a defect in neurite extension similar to that seen in granule cells (Rakic and Sidman, 1973; Roffler-Tarlov et al., 1996), and this may reflect some degree of cell body ectopia. Second, death of these neurons takes place during the second and third postnatal weeks of development (Roffler-Tarlov et al., 1996). Although the timing of this death is shifted by 1 week from what we observe, it appears that late-generated dopaminergic neurons are preferentially affected by the mutation (Bayer et al., 1995). This effect may be similar to deleterious effects on late-born large neurons in the cerebellum (see below).

\section{Implications for normal cerebellar development}

Our characterization of large neuron disruptions in weaver cerebella forces the consideration of a new component to early cerebellar development. Previous researchers (Altman and Bayer, 1985a,b,c) have suggested that DCN neurons follow a lateral-tomedial pattern of migration after their birth, whereas Purkinje cells migrate more radially toward the pial surface; both of these cell types originate in the ventricular epithelium of the fourth ventricle. The nature of the movements that lead to the fusion of the initially separate bilateral anlage, however, are totally unknown. Our work provides the first evidence for a lateral-tomedial component of Purkinje cell movement during normal cerebellar development; this movement forms a likely component of the fusion.

Some Purkinje cells and DCN neurons seem fully capable of completing their migration to the midline of the weaver cerebel- 
lum, just as some granule cells are capable of completing their radial migration (Herrup and Trenkner, 1987). This incomplete penetrance of the migration phenotype hints that there may be separate waves of migration: some are GIRK2 independent and early, whereas others are GIRK2-dependent and late. This interpretation fits very well with the studies of Altman and Bayer (1985c); earlier migrations of Purkinje cells might be radial, and potentially unaffected by GIRK2, whereas later migrations might follow a course similar to that of the migrating DCN neurons and therefore be indistinguishable from them as judged by anatomical observations. Consistent with this idea, the later-born Purkinje cells and DCN neurons are found to populate more medial regions of the cerebellum (Taber Pierce, 1975; Altman and Bayer, 1985c).

The relatively late timing of large neuron death suggests that there may be a critical period of target dependency beginning sometime during the third postnatal week. Virtually all movement of postmitotic DCN neurons to their positions in the deep nuclei and of Purkinje cells to the cortex is completed by birth (Altman and Bayer, 1985a,b,c). Labeling studies have demonstrated that DCN neurons make contact with rostral brain regions during late embryogenesis. The exact timing of the establishment of communication between the DCN neurons and Purkinje cells is not known.

\section{GIRK2 function in the developing cerebellum}

The question arises as to how this scenario of cerebellar cell movements in weaver is related to the known molecular defect, which is a point mutation in the pore region of the inwardly rectifying potassium channel subunit GIRK2. Although adult expression of girk2 mRNA is confined to granule cells (Patil et al., 1995; Kofugi et al., 1996; Slesinger et al., 1996) and possibly low levels in the DCN (Liao et al., 1996), in situ hybridization reveals gene expression in the ventricular zone of the fourth ventricle as early as embryonic day 14.5 (Kofugi et al., 1996), and Purkinje cells have been shown to express girk2 as late as P7 (Slesinger et al., 1996). One possibility is that migrating neurons "sense" an external electrochemical gradient and use it as a guide for directing their movements. Impaired conduction through channels containing GIRK2 subunits may impair the ability of the affected neuron to read this gradient, resulting in a failure of migration. In this way, it is possible that girk2 expression is necessary for the proper migration of subsets of all three major cerebellar cell types: granule cells, Purkinje cells, and DCN neurons. Our data support this argument for Purkinje cells and DCN neurons, and Smeyne and Goldowitz (1989) demonstrated that the first difference in granule cell numbers, noted at $\mathrm{P} 2$, coincides with the beginning of migration from the external to the internal granule cell layer. Another possibility is that the GIRK2 channel protein is needed for the mechanics of migration itself. Signaling cascades triggered by alterations in membrane potential might be important for directing neuron movement. Additional studies are needed to clarify the role of GIRK2 in this process.

\section{REFERENCES}

Altman J, Anderson WJ (1972) Experimental reorganization of the cerebellar cortex. I. Morphological effects of elimination of all microneurons with prolonged x-irradiation started at birth. J Comp Neurol 146:355-406.

Altman J, Bayer S (1985a) Embryonic development of the rat cerebellum. I. Delineation of the cerebellar primordium and early cell movements. J Comp Neurol 231:1-26.
Altman J, Bayer S (1985b) Embryonic development of the rat cerebellum. II. Translocation and regional distribution of the deep neurons. J Comp Neurol 231:27-41.

Altman J, Bayer S (1985c) Embryonic development of the rat cerebellum. III. Regional differences in the time of origin, migration and settling of Purkinje cells. J Comp Neurol 231:42-65.

Armstrong DM, Schild RF (1978a) An investigation of the cerebellar cortico-nuclear projections in the rat using an autoradiographic tracing method. I. Projection from the vermis. Brain Res 141:1-19.

Armstrong DM, Schild RF (1978b) An investigation of the cerebellar corticonuclear projections in the rat using an autoradiographic tracing method. II. Projections from the hemisphere. Brain Res 141:235-249.

Bayer SA, Altman J (1996) 3-D reconstructions of fiber partitioning and lobulation in the cerebellar vermis and hemisphere. Soc Neurosci Abstr 23:15.

Bayer SA, Wills KV, Triarhou LC, Verina T, Thomas JD, Ghetti B (1995) Selective vulnerability of late-generated dopaminergic neurons of the substantia nigra in weaver mutant mice. Proc Natl Acad Sci USA 92:9137-9140.

Blatt GJ, Eisenman LM (1985) A qualitative and quantitative light microscopic study of the inferior olivary complex of normal, reeler, and weaver mutant mice. J Comp Neurol 232:117-128.

Caddy KWT, Biscoe TJ (1979) Structural and quantitative studies on the normal C3H and lurcher mutant mouse. Philos Trans R Soc Lond [Biol] 287:167-201.

Clarke PGH, Cowan WM (1976) The development of the isthmo-optic tract in the chick, with special reference to the occurrence and correction of developmental errors in the location and connections of the isthmo-optic neurons. J Comp Neurol 167:143-164.

Hendry IA (1976) A method to correct adequately for the change in neuronal size when estimating neuronal numbers after nerve growth factor treatment. J Neurocytol 5:337-349.

Herrup K (1986) Cell lineage relationships in the development of the mammalian CNS. III. Role of cell lineage in regulation of Purkinje cell number. Dev Biol 115:148-154.

Herrup K, Mullen RJ (1979) Regional variation and absence of large neurons in the cerebellum of the staggerer mouse. Brain Res 172:1-12.

Herrup K, Sunter K (1986) Cell lineage dependent and independent control of Purkinje cell number in the mammalian CNS: further quantitative studies of lurcher chimeric mice. Dev Biol 117:417-427.

Herrup K, Trenkner E (1987) Regional differences in cytoarchitecture of the weaver cerebellum suggest a new model for weaver gene action. Neuroscience 23:871-885.

Herrup K, Wetts R, Diglio TJ (1984) Cell lineage relationships in the development of the mammalian CNS. II. Bilateral independence of CNS clones. J Neurogenet 1:275-288.

Jansen J, Brodal A (1940) Experimental studies on the intrinsic fibers of the cerebellum. II. The cortico-nuclear projection. J Comp Neurol 73:267-321.

Kofugi P, Hofer M, Millen KJ, Millonig JH, Davidson N, Lester HA, Hatten ME (1996) Functional analysis of the weaver mutant GIRK2 $\mathrm{K}^{+}$channel and rescue of weaver granule cells. Neuron 16:941-952.

Korneliussen HK (1968) On the morphology and subdivision of the cerebellar nuclei of the rat. J Hirnforsch 10:109-122.

Liao YJ, Jan YN, Jan LY (1996) Heteromultimerization of G-proteingated inwardly rectifying $\mathrm{K}^{+}$channel proteins GIRK1 and GIRK2 and their altered expression in weaver brain. J Neurosci 16:7137-7150.

Linden R (1994) The survival of developing neurons: a review of afferent control. Neuroscience 58:671-682.

Mariani J, Benoit P, Hoang MD, Thomson MA, Delhaye-Bouchaud N (1990) Extent of multiple innervation of cerebellar Purkinje cells by climbing fibers in adult $x$-irradiated rats: comparison of different schedules of irradiation during the first postnatal week. Dev Brain Res 57:63-70.

Millen KJ, Wurst W, Herrup K, Joyner AL (1994) Abnormal embryonic cerebellar development and patterning of postnatal foliation in two mouse Engrailed-2 mutants. Development 120:695-706.

Oppenheim R (1991) Cell death during development of the nervous system. Annu Rev Neurosci 14:453-501.

Patil N, Cox DR, Bhat D, Faham M, Myers RM, Peterson AS (1995) A potassium channel mutation in weaver mice implicates membrane excitability in granule cell differentiation. Nature Genet 11:126-129.

Rakic P, Sidman RL (1973) Sequence of developmental abnormalities leading to granule cell deficit in cerebellar cortex of weaver mutant mice. J Comp Neurol 152:103-132. 
Rezai Z, Yoon CH (1972) Abnormal rate of granule cell migration in the cerebellum of "weaver" mutant mice. Dev Biol 29:17-26.

Roffler-Tarlov S, Martin B, Graybiel AM, Kauer JS (1996) Cell death in the midbrain of the murine mutation weaver. J Neurosci 16:1819-1826.

Sekiguchi M, Nowakowski RS, Nagato Y, Tanaka O, Guo H, Madoka M, Abe H (1995) Morphological abnormalities in the hippocampus of the weaver mutant mouse. Brain Res 696:262-267.

Shirasaki R, Tamada A, Katsumata R, Murakami F (1995) Guidance of cerebellofugal axons in the rat embryo: directed growth toward the floor plate and subsequent elongation along the longitudinal axis. Neuron 14:961-972.

Sidman RL, Green MC (1970) "Nervous", a new mutant mouse with cerebellar disease. In: Les mutants pathologiques chez l'animal (Sobourdy M, ed), pp 69-79. Paris: Editions du Centre National de la Recherche Scientifique.
Slesinger PA, Patil N, Liao YJ, Jan YN, Jan LY, Cox DR (1996) Functional effects of the mouse weaver mutation on $G$ protein-gated inwardly rectifying $\mathrm{K}^{+}$channels. Neuron 16:321-331.

Smeyne RJ, Goldowitz D (1989) Development and death of external granular layer cells in the weaver mouse cerebellum: a quantitative study. J Neurosci 9:1608-1620.

Taber Pierce E (1975) Histogenesis of the deep cerebellar nuclei in the mouse: an autoradiographic study. Brain Res 95:503-518.

Triarhou LC, Norton J, Ghetti B (1987) Anterograde transsynaptic degeneration in the deep cerebellar nuclei of Purkinje cell degeneration ( $p c d)$ mutant mice. Exp Brain Res 66:577-588.

Weimer WR, Lane PW, Sidman RL (1982) Vibrator ( $v b)$ : a spinocerebellar system degeneration with autosomal recessive inheritance in mice. Brain Res 1982:357-364. 